\title{
Prognostic Factors for Survival after Resection of Liver Metastases from Colorectal Cancer: A Single Institution Analysis of 655 Cases
}

\author{
Sorin Alexandrescu ${ }^{1 *}$, Andrei Diaconescu ${ }^{1 *}$, Narcis Octavian Zarnescu ${ }^{2}$, Zenaida lonel ${ }^{1}$, Cristian Zlate ${ }^{1}$, \\ Doina Hrehoret ${ }^{1}$, Vladislav Brasoveanu ${ }^{1}$, Razvan Grigorie ${ }^{1}$, Florin Botea ${ }^{1}$, Dana Tomescu ${ }^{3}$, Gabriela Droc ${ }^{3}$, \\ Ruxandra Fota ${ }^{3}$, Daniela Ungureanu ${ }^{3}$, Adina Croitoru4, Mirela Boros ${ }^{5}$, Mugur Grasu' ${ }^{5}$, Radu Dumitru ${ }^{5}$, \\ Mihai Toma ${ }^{5}$, Vlad Herlea ${ }^{6}$, Mihnea lonescu ${ }^{1}$, Irinel Popescu ${ }^{1}$
}

\author{
"Dan Setlacec" Centre of General Surgery and Liver Transplantation \\ Fundeni Clinical Institute, Bucharest, Romania \\ ${ }^{2}$ Second Department of Surgery, University Emergency Hospital Bucharest, Romania \\ ${ }^{3}$ Centre of Anaesthesiology and Intensive Care, Fundeni Clinical Institute, Bucharest, Romania \\ ${ }^{4}$ Department of Oncology, Fundeni Clinical Institute, Bucharest, Romania \\ ${ }^{5}$ Department of Radiology, Fundeni Clinical Institute, Bucharest, Romania \\ ${ }^{6}$ Department of Pathology, Fundeni Clinical Institute, Bucharest, Romania
}

\section{ABSTRACT}

Backgound: Several clinical risk factors for patients undergoing liver resection for colorectal liver metastases were suggested. The purpose of the study was to evaluate the prognostic factors for survival after resection of liver metastases from colorectal cancer in a high volume center for both hepatobiliary and colorectal surgery.

Methods: We completed a retrospective analysis on 655 consecutive patients with liver resection for colorectal cancer metastasis operated in our centre between April 1996 and March 2016. Preoperative, intraoperative, pathologic, and outcome data for patients undergoing liver resection for metastatic colorectal were examined. Univariate analysis followed by multivariate Cox regression analysis was performed in order to identify the risk factors associated with prognostic factors related to survival.

Results: There were 371 men (56.65\%) and 284 women (43.35\%), with a median age of 60 year-old (range 24 to 84$)$. The primary tumor location was colon in 454 cases $(69.31 \%)$ and rectum for 201 cases (30.69\%). Synchronous tumors were resected in 353 cases $(53.72 \%)$. The surgical mortality rate was $2.95 \%$. The 5 -year survival rate was $29.2 \%$, and the 10 -year survival rate was $17.1 \%$. Four factors were found to be significant independent predictors of poor long-term outcome by multivariate analysis: bilobar liver metastasis $(p=0.003)$ with HR $1.653(95 \% \mathrm{Cl} 1.180-2.316)$, non-R0 resection $(p<0.001)$ with $\mathrm{HR} 6.066(95 \% \mathrm{Cl}$ $3.508-10.489)$, N2 lymph node stage $(p=0.007)$ with HR $1.528(95 \% \mathrm{Cl} 1.125-2.075)$ and lack of adjuvant chemotherapy $(\mathrm{p}=0.046)$ with HR $1.703(95 \% \mathrm{Cl} 1.009-2.873)$.

Conclusion: The independent prognostic factors for poor OS were both clinico-pathologic and therapeutic. In patients with good prognostic factors, an appropriate onco-surgical treatment is able to significantly prolong survival. In patients with poor prognostic factors, prognosis is mainly influenced by tumor biology and the benefit from current therapies is still modest.

Key words: colorectal liver metastasis, liver resection, adjuvant therapy, survival, risk factors
Corresponding author: Irinel Popescu, MD, PhD, FACS, FEBS, Professor of Surgery "Dan Setlacec" Centre of General Surgery and Liver Transplantation Fundeni Clinical Institute, Bucharest Soseaua Fundeni, No. 258, sector 2 Bucharest, Romania, 022528 Tel/Fax: (+4)0213180417 E-mail: irinel.popescu220@gmail.com

\footnotetext{
* These authors equally contributed to this paper
}

Abbreviations: CLMs - colorectal liver metastases OS - overall survival

Received: 17.11.2017 Accepted: 10.12 .2017 


\section{INTRODUCTION}

Colorectal cancer (CRC) is the third malignancy among the adult patients, representing the third cause of cancer-related death (1). The main cause of decease in these patients is the metastatic disease, with more than two thirds of them presenting liver metastases at the time of death (2).

In present, the treatment of these patients is multimodal, involving liver resection, ablative therapies, systemic or loco-regional cytotoxic treatment, targeted therapies and immunotherapy.

A recent study revealed that the only independent factors associated with improved survival in patients with metastatic colorectal cancer were the increased number of patients undergoing liver resection for liver metastases and the use of new oncologic drugs (e.g. Oxaliplatin, Irinotecan, Bevacizumab, Cetuximab, Panitumumab)(2). However, the 5-year overall survival rates achieved by liver resection in patients with colorectal cancer liver metastases (CLMs) vary on a large scale among centres, ranging between $25 \%$ and $58 \%$ (3-9). One of the explanations for these results is the difference between centres regarding the selection criteria for hepatectomy. Another explanation may be the differences in indications of oncologic therapies in different centres (e.g. preoperative chemotherapy is routinely recommended in some centres, while others offer this treatment only in patients with more advanced disease).

Because the factors related to the primary tumor, metastases and response to chemotherapy largely differ between patients, in order to perform a comprehensive comparison it is advisable to compare the patients with similar prognosis after liver resection.

Therefore, identification of the factors that significantly influence survival in patients who underwent liver resection of CLMs is tremendously important. This allows stratifying patients based on their prognostic factors, eventually leading to a tailored therapy for each group. Thus, in patients with poor prognostic factors, an aggressive chemotherapy regimen could improve the life-expectancy. Moreover, identification of the prognostic factors may be useful in stratifying patients for clinical trials, in order to compare the advantages offered by several therapies (such as ablative therapies or adjuvant and neo-adjuvant chemotherapy) in patients with similar prognosis.

Such factors have been evaluated in many other studies, but the results were not uniform (10-13). Taking into account the results reported by previous studies, this paper analyzed a large experience of liver resection for CLMs at a tertiary referral centre, in order to identify the factors that significantly influence overall survival in such patients. Finally, it was attempted to create a clinical scoring system able to stratify patients with CLMs for liver resection and clinical studies.

\section{MATERIAL AND METHODS}

All the patients with CLMs who were operated with curative intent in "Dan Setlacec" Centre of General Surgery and Liver Transplantation from Fundeni Clinical Institute, Bucharest between April 1996 and March 2016 were included in this study.

Selection criteria for liver resection were: medical fitness for surgery, anticipated ability to perform a complete resection (RO) of CLMs, the volume of functional liver remnant (FLR) after hepatectomy more than 25-30\% of total liver volume, the ability to perform a complete resection of extrahepatic disease in patients with concomitant extrahepatic metastases (EHMs).

Preoperative assessment of patients included abdominal and pelvic contrast-enhanced CT scan or MRI, at least chest X-ray (until 2006) and thorax CT scan (since 2007), as well as PET/CT when suspicion of extrahepatic disease were raised (since 2014). When symptoms raised the suspicion of bone metastases or central nervous system metastases, bone scintigraphy and head CT scan, respectively, was performed. In patients operated as emergency due to the complications of the primary tumor, when preoperative CT scan was not performed, intraoperative exploration was the method for identification of CLMs. All these patients had preoperative chest $\mathrm{X}$-ray and underwent postoperative $\mathrm{CT}$ scan during the first 3 months after primary resection.

The data regarding clinical characteristics of the patients, the characteristics of the primary tumor and metastases were identified from hospital charts, discharge letters from other hospitals (in patients undergoing primary tumor resection in other hospitals), Liver resection database, and Operation database of "Dan Setlacec" Centre of General Surgery and Liver Transplantation from Fundeni Clinical Institute.

CLMs were considered synchronous when they were detected before or during the operation addressed to the primary tumor, in patients who underwent preoperative CT scan. When CT scan was not performed preoperatively, if the metastases were detected at the CT scan performed during the first 3 months postoperatively, they have been considered also synchronous CLMs. Bilobar distribution was considered only in patients presenting two or more CLMs and not in patients with a single metastasis. The 
number and maximum size of metastases are based on the pathologic report after liver resection. CLMs were considered initially unresectable when the evaluation (in our centre) of imaging performed at the time of their diagnosis revealed that a complete resection would not spare at least $25-30 \%$ of total liver volume. Liver resection was considered a major hepatectomy when 3 or more liver segments were entirely resected. The resection was defined R1 when pathologic examination revealed microscopic involvement of the cut-surface of the specimen and R2 when pathology report disclosed macroscopic involvement of the cut-surface of the specimen or when the surgeon left in place macroscopic tumoral tissue (either hepatic or extrahepatic).

The following data were evaluated: age, gender, location of the primary tumor, pathology data of the colorectal lesion $(T, N)$, timing of CLMs detection (synchronous vs. metachronous), maximum metastases diameter, number of CLMs, uni/bilobar distribution, initial resectability, surgical details (type and extent of liver resection), completeness of resection ( $R O$ vs R1/R2), presence of extrahepatic disease, CEA and CA19-9 levels, the use of neo-adjuvant chemotherapy and adjuvant chemotherapy, as well as the mortality and overall survival rates.

Postoperative mortality was defined as the decease of the patient during hospitalization or within the first 30 days after hepatectomy.

The overall survival (OS) was defined as the interval between the liver resection and the last follow-up or the date of patient's death.

Patients who died postoperatively were excluded from survival analysis, as well as the patients who were lost at follow-up.

Follow-up was performed by personal contact with the patient, the patient's family, or the attending oncologist.

In some patients, reliable data were not available for certain parameters. That lacking data occurs mainly in patients operated for their primary tumors in other hospitals or those who were not followed in our institution.

Continuous data is presented as median. Categorical data is presented as proportions. Using the Kaplan-Meier method differences in survival were examined using the log-rank test. To evaluate independent risk factors associated with OS, multivariate Cox proportional hazards regression analysis was used. In multivariate analysis were included the parameters that were correlated at univariate analysis with $p$ value $<0.10$ (except the CEA and
CA $19-9$ level - missing data in more than $66 \%$ patients).

After identification of independent poor prognostic factors, the patients were divided in three groups according to the number of these parameters present in each patient. The OS rates for the three groups were compared with log-rank test, to assess if these factors are able to accurately stratify the patients regarding their prognosis.

A two-sided $p$ value lower than 0.05 was considered to be significant.

Statistical analysis was performed using SPSS software package, version 20 (SPSS Inc, Chicago, IL).

\section{RESULTS}

Between April 1996 and March 2016, 678 patients with CLMs underwent liver resection.

The postoperative mortality rate was $2.94 \%$ (20/678 patients). These patients were excluded from survival analysis, as well as the patients who were lost at follow-up (3 patients). Thus, 655 patients were analyzed for identification of prognostic factors for long-term survival.

\section{Demographics}

There were 371 men and 284 women, with a median age of 60 year-old (range 24 to 84 ); 443 were younger than 65 year-old.

At the time of last follow-up, 479 patients had died; median time from liver resection to the last follow-up 26.2 months (range 1.2 to 143.2 months). One hundred seventy six patients were alive at the last follow-up (median 55.8 months, range 19 to 197.6 months).

\section{Primary tumor characteristics}

The primary tumor was located in the rectum in 201 (30.69\%) patients, in the right colon in $20.12 \%$ patients and in the left colon in $49.19 \%$ patients. The majority of patients (387 - 79.79\%) presented with T3 colorectal cancer, while T4 primaries were present in 64 (13.20\%) patients and $\mathrm{T} 2$ primaries in 34 (7.01\%); missing data in 170 patients. Four or more regional lymph nodes present metastases (N2) in 149 patients, while 317 (68.02\%) patients presented 0-3 metastatic lymph nodes (NO/N1); missing data in 190 patients. At the time of primary tumor diagnosis $353(53.72 \%)$ patients presented CLMs (synchronous metastases), while in the other patients (302 - 46.28\%) the metastases developed metachronous. 


\section{CLMs features}

The median size of liver metastases was $3.5 \mathrm{~cm}$ (range 0.3 to $24 \mathrm{~cm}$ ); $437(67.33 \%$ ) patients presented CLMs smaller than $5 \mathrm{~cm}$ and 212 (32.67\%) patients with CLMs larger than $5 \mathrm{~cm}$ (missing data in 6 patients). The number of liver metastases was 4 or more in 82 (12.51\%) patients. The metastases presented bilobar involvement of the liver in 181 (27.77\%) patients and 471 (72.23\%) patients have monolobar CLMs; missing data in 3 patients. In 44(6.72\%) patients, CLMs were deemed as initially unresectable at the time of their diagnosis, being rendered to resectability by one of the following onco-surgical strategies: resection after portal vein ligation (10 patients), „two-stage" liver resection (5 patients), resection after down-sizing chemotherapy (4 patients) or combination of liver resection and ablation (23 patients). Liver metastases were associated with extrahepatic disease in 69 (10.53\%) patients (colorectal hepatic and extrahepatic metastases CHEHMs).

\section{Liver resection}

Non-anatomic hepatectomy was performed in 479 (73.12\%) patients, anatomical resection was performed in $133(20.30)$ patients, while in $43(6.58 \%)$ patients an anatomic liver resection was associated with a nonanatomical procedure. A major liver resection was performed in $123(18.78 \%)$ patients. A tumor free resection margin was achieved in 620 (94.65\%) patients.

\section{Chemotherapy}

Preoperative (neo-adjuvant) chemotherapy was used before liver resection in 169 (25.92\%) patients, while 483 (74.08\%) patients underwent up-front surgery; missing data in 3 patients. Data about postoperative chemotherapy regimens were available in 287 (92.58\%) patients, while 23 patients did not receive postoperative chemotherapy. For 345 patients the data regarding chemotherapy were not reliable (patients who were oncologic followed in other institutions and unable to mention the chemotherapy regimen).

\section{Tumor markers}

The median level of CEA was $18 \mathrm{ng} / \mathrm{ml}$ (range 1 to $1264 \mathrm{ng} / \mathrm{ml}$ ), with lacking data in 430 patients. The preoperative level of CA 19-9 was available in 190 patients, with median level $31 \mathrm{U} / \mathrm{ml}$ (range 0 to 1200). Because the level of tumor markers was not available in more than $66 \%$ of patients, these parameters were excluded from multivariate analysis.

For the entire group the 1-, 3-, 5- and 10-year OS rates were $87.2 \%, 48.5 \%, 29.2 \%$ and $17.1 \%$, respectively, with 35.5 months median survival. To assess if the long-term outcomes changed over the time, the entire period (2006-2016) was divided in 3 consecutive time periods (1996-2005, 2006-2010 and 2011-2016). Comparison of OS rates achieved in each of these time periods revealed that survival constantly improved over the time, with median survival of 29.4, 33.9 and 39.4 months, respectively for periods 1996-2005, 2006-2010 and 2011-2016 ( $p$ value $=0.001)$ (fig. 1) .

\section{Univariate predictors for long-term survival}

No one of the demographic parameters (age or gender) has a significant impact on OS after resection of CLMs ( $p$ value> 0.05) (table 1).

Among the factors related to the primary tumor, the primary tumor location (rectum vs. colon, or right colon vs. left colon) and the timing of CLMs development (synchronous vs. metachronous) had no significant impact on OS ( $p$ value> 0.05) (table 1). The grade of parietal wall penetration by the primary tumor significantly influences the OS, with $T 2$ patients achieving the highest survival rates and T4 the lowest survival rates ( $p$ value $=0.034)$ (fig. 2). Metastatic involvement of 4 or more lymph nodes (N2) was significantly associated with lower OS than in NO/N1 patients ( $p$ value < 0.00001) (fig. 3).

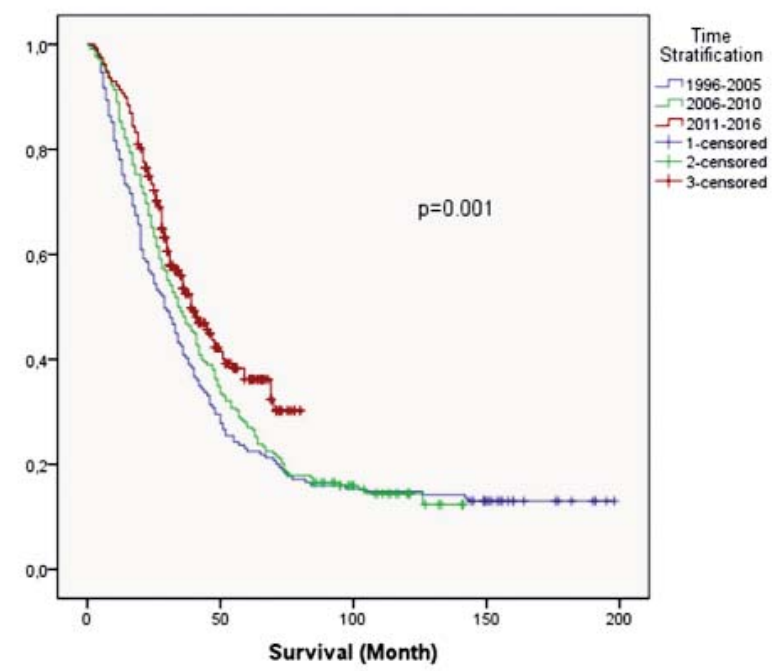

Figure 1 - Comparative overall survival related to period of hepatectomy 
Table 1 - Univariate predictors for OS in patients undergoing hepatectomy for CLMs

\begin{tabular}{|c|c|c|c|c|}
\hline Variable & Patients number (\%) & 5-year OS & Median survival (months) & $\mathrm{p}$ value \\
\hline Age & & & & 0.145 \\
\hline$>65$ & $212(32.36 \%)$ & $26.1 \%$ & 33 & \\
\hline$<65$ & $443(67.63 \%)$ & $29.9 \%$ & 36 & \\
\hline Gender & & & & 0.363 \\
\hline $\mathrm{F}$ & $284(43.35 \%)$ & $27.1 \%$ & 33 & \\
\hline $\mathrm{M}$ & $371(56.65 \%)$ & $30 \%$ & 37 & \\
\hline Primary tumor location & & & & 0.853 \\
\hline Rectum & $201(30.69 \%)$ & $28.2 \%$ & 36 & \\
\hline Colon & $454(69.31 \%)$ & $28.9 \%$ & 36 & \\
\hline Colon location & & & & 0.957 \\
\hline Right colon & $20.12 \%$ & $33.1 \%$ & 34 & \\
\hline Left Colon & $49.19 \%$ & $28.8 \%$ & 37 & \\
\hline T status & & & & 0.034 \\
\hline $\mathrm{T} 2$ & $34(7.01 \%)$ & $39.5 \%$ & 48 & \\
\hline T3 & $387(79.79 \%)$ & $28.1 \%$ & 35 & \\
\hline T4 & $64(13.20 \%)$ & $22.7 \%$ & 33 & \\
\hline N status & & & & $<0.00001$ \\
\hline NO-N1 & $317(68.02 \%)$ & $33.7 \%$ & 42 & \\
\hline N2 & $149(31.97 \%)$ & $19 \%$ & 27 & \\
\hline CLMs development & & & & 0.276 \\
\hline Synchronous & $353(53.72 \%)$ & $27.2 \%$ & 34 & \\
\hline Metachronous & $302(46.28 \%)$ & $30.3 \%$ & 36 & \\
\hline Maximum CLMs diameter & & & & 0.038 \\
\hline$<5 \mathrm{~cm}$ & $437(67.33 \%)$ & $30.8 \%$ & 39 & \\
\hline$\geq 5 \mathrm{~cm}$ & $212(32.67 \%)$ & $24.3 \%$ & 30 & \\
\hline Number of metastases & & & & $<0.00001$ \\
\hline $1-3$ & $573(53.89 \%)$ & $31.4 \%$ & 37 & \\
\hline$\geq 4$ & $82(12.51 \%)$ & $7.4 \%$ & 26 & \\
\hline Location of CLMs & & & & 0.001 \\
\hline Unilobar & $471(72.23 \%)$ & $31.8 \%$ & 39 & \\
\hline Bilobar & $181(27.77 \%)$ & $19.9 \%$ & 30 & \\
\hline Initial resectability & & & & 0.333 \\
\hline Initially resectable & $611(93.28 \%)$ & $29.2 \%$ & 36 & \\
\hline Initially unresectable & $44(6.72 \%)$ & $18.8 \%$ & 31 & \\
\hline Type of liver resection & & & & 0.889 \\
\hline Anatomical & $133(20.30)$ & $26.8 \%$ & 29 & \\
\hline Non-anatomical & $479(73.12 \%)$ & $30.3 \%$ & 37 & \\
\hline Combined & $43(6.58 \%)$ & $14.1 \%$ & 27 & \\
\hline Extent of hepatectomy & & & & 0.046 \\
\hline Minor resection & $532(81.22 \%)$ & $29.8 \%$ & 36 & \\
\hline Major resection & $123(18.78 \%)$ & $23.4 \%$ & 30 & \\
\hline Completeness of resection & & & & $<0.00001$ \\
\hline Ro & $620(94.65 \%)$ & $30.2 \%$ & 37 & \\
\hline $\mathrm{R} 1 / \mathrm{R} 2$ & $35(5.35 \%)$ & $0 \%$ & 13 & \\
\hline Adjuvant chemotherapy & & & & 0.030 \\
\hline Yes & $287(92.58 \%)$ & $29.2 \%$ & 40 & \\
\hline No & $23(7.42 \%)$ & $17.9 \%$ & 28 & \\
\hline Neo-adjuvant chemotherapy & & & & 0.220 \\
\hline Yes & $169(25.92 \%)$ & $24.5 \%$ & 34 & \\
\hline No & $483(74.08 \%)$ & $30 \%$ & 36 & \\
\hline CEA & & & & 0.008 \\
\hline $\mathrm{CEA}>5.2 \mathrm{ng} / \mathrm{ml}$ & $176(78.22 \%)$ & $30.4 \%$ & 36 & \\
\hline $\mathrm{CEA}<5.2 \mathrm{ng} / \mathrm{ml}$ & $49(21.78 \%)$ & $51.9 \%$ & 64 & \\
\hline CA19-9 & & & & 0.025 \\
\hline $\mathrm{CA} 19-9<37 \mathrm{U} / \mathrm{ml}$ & $107(56.31 \%)$ & $41.9 \%$ & 48 & \\
\hline CA19-9 >37 U/ml & $83(43.69 \%)$ & $31.7 \%$ & 36 & \\
\hline Extrahepatic metastases & & & & $<0.0001$ \\
\hline Yes & $69(10.53 \%)$ & $9.2 \%$ & 19 & \\
\hline No & $586(89.47 \%)$ & $30.9 \%$ & 39 & \\
\hline
\end{tabular}




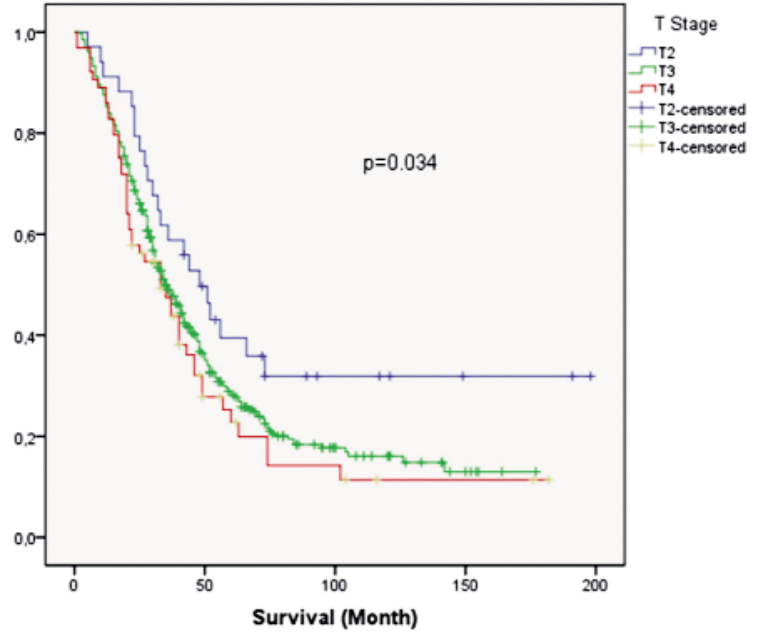

Figure 2 - Comparative overall survival between T2 vs T3 vs T4

Apart of the initial resectability of CLMs which does not significantly influence survival ( $p$ value $=0.333$ ), all the other factors relatedwith CLMs significantly impact OS (table 1). Thus, CLMs larger than $5 \mathrm{~cm}$ were associated with significantly poorer prognosis than those smaller than $5 \mathrm{~cm}$ ( $p$ value $=0.038)(f i g .4)$. Patients with 4 or more CLMs have a significantly lower OS than those with up to 3 CLMs ( $p$ value $<0.00001$ ) (fig. 5). Bilobar distribution of CLMs is significantly associated with decreased survival when compared with unilobar location of metastases $(p$ value $=0.001)$ (fig. 6).

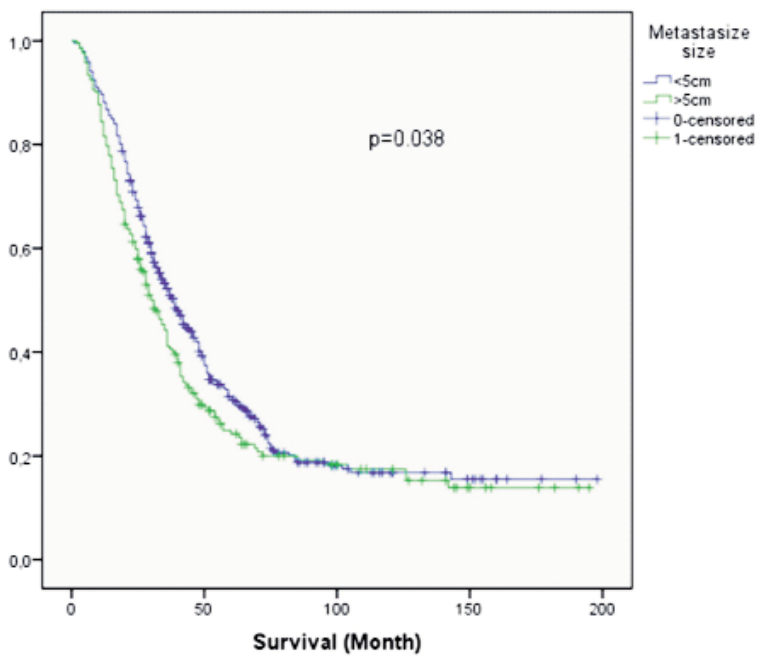

Figure 4 - Comparative overall survival between metastasize size

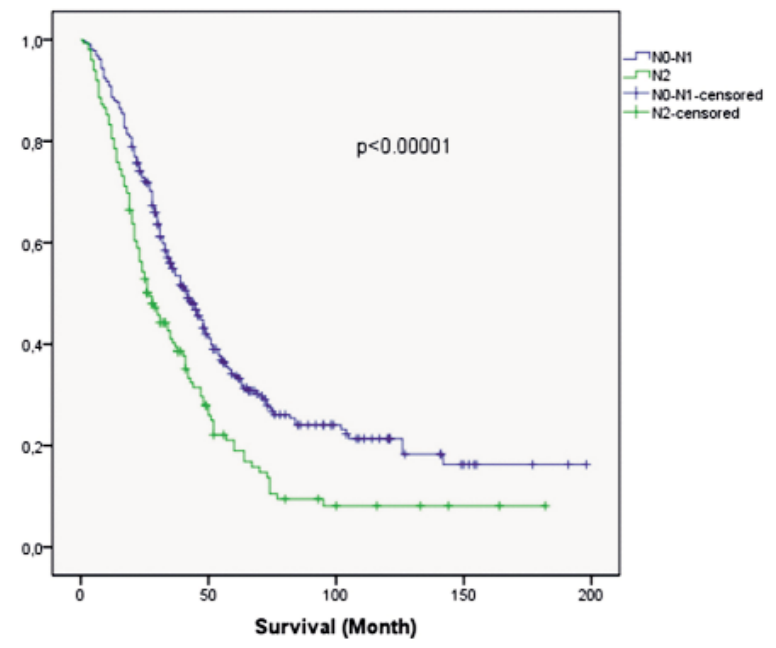

Figure 3 - Comparative overall survival between N0-N1 vs N2

Surgical factors that significantly improved OS were minor hepatectomy (higher survival rates than major hepatectomy $-p$ value $=0.046$ ) (fig. 7 ) and the ability to perform RO resection (significantly better OS than in patients who underwent $R 1 / R 2$ resections $-p$ value $<$ 0.00001) (fig. 8). The type of liver resection (anatomical vs. non-anatomical vs. Combined hepatectomy) did not influence in a significant manner the long-term outcomes ( $p$ value $=0.889$ ) (table 1$)$.

While neo-adjuvant chemotherapy did not improved significantly OS of patients undergoing liver resection for CLMs ( $p$ value $=0.220)($ table 1$)$, adjuvant chemotherapy

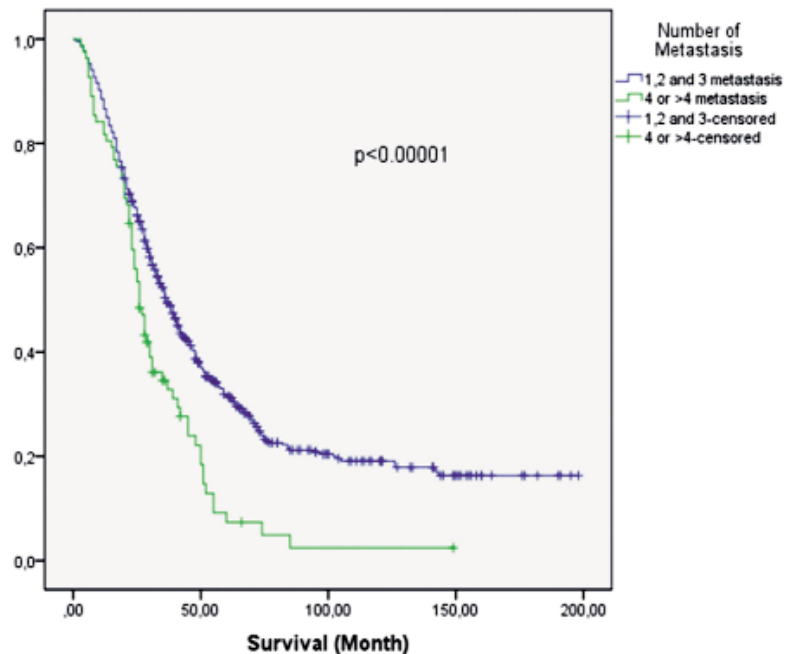

Figure 5 - Comparative overall survival between number of metastasis 


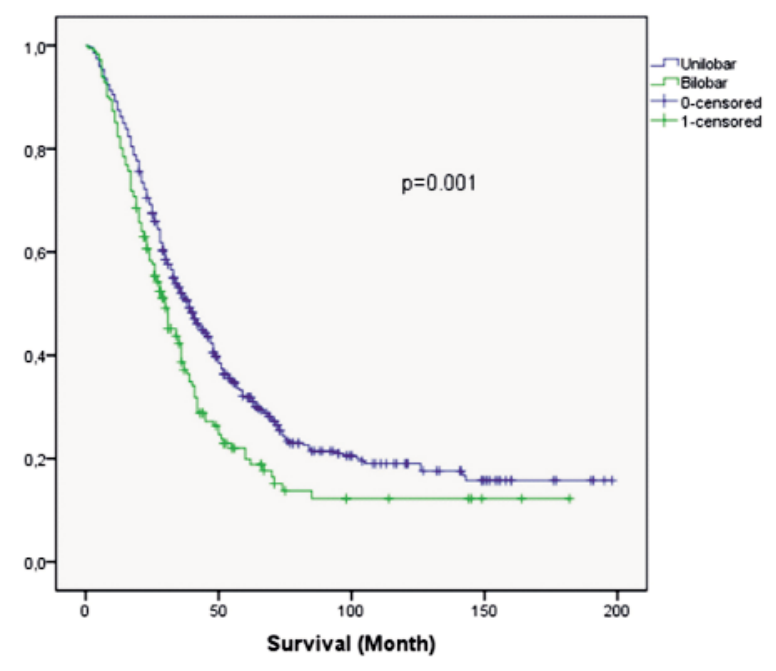

Figure 6 - Comparative overall survival between Location of CLMs (bilobar vs unilobar)

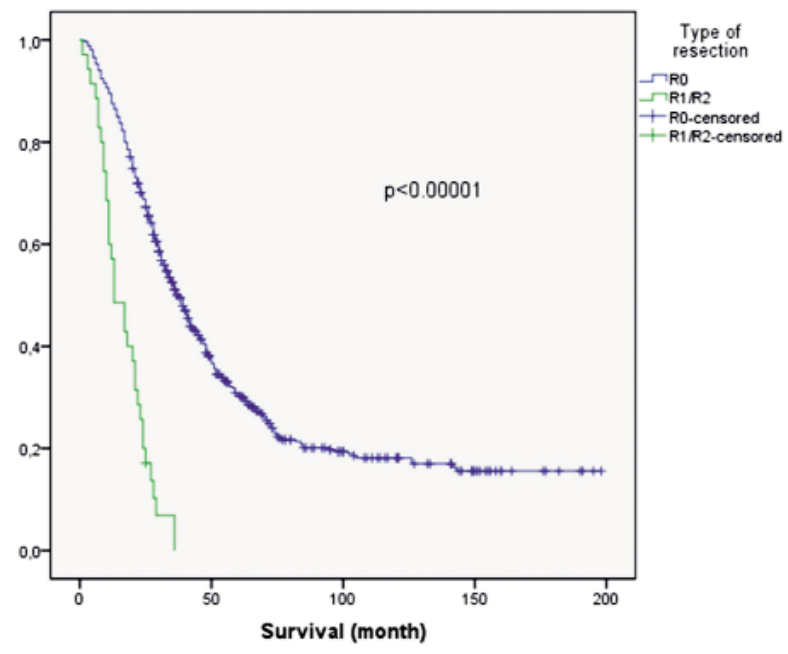

Figure 8 - Comparative overall survival between R0 vs R1/R2

significantly prolonged survival compared with patients who did not receive postoperative chemotherapy ( $p$ value $=0.030)$ (fig. 9).

In univariate analysis, increased preoperative levels of both CEA and CA 19-9 were predictors for poor outcome ( $p$ value< 0.05 ) (fig. 10 and 11).

The concomitant presence of hepatic and extrahepatic metastases significantly decreased OS compared with patients with liver-limited metastases ( $p$ value < 0.0001) (fig. 12).

\section{Multivariate analysis of predictors for $O S$}

To determine the independent predictors of OS

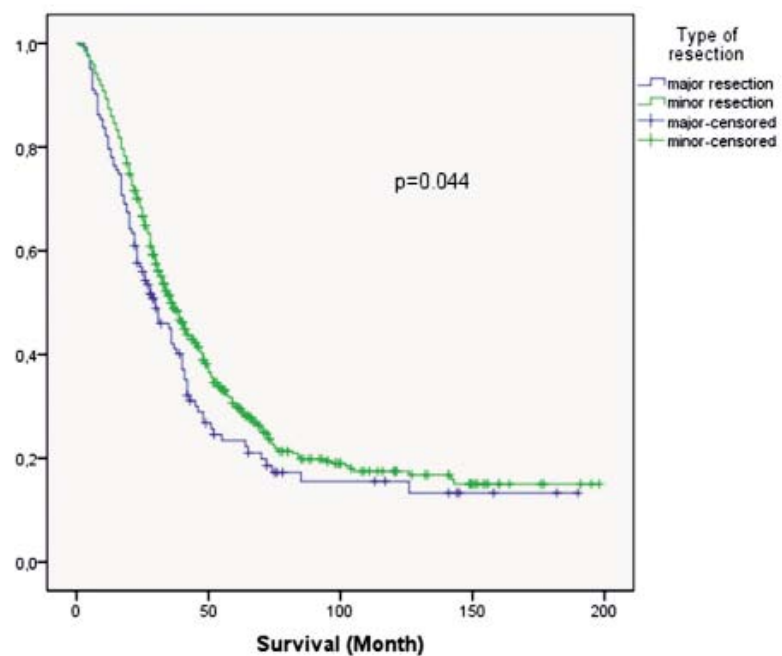

Figure 7 - Comparative overall survival between type of resection (major resection vs minor resection)

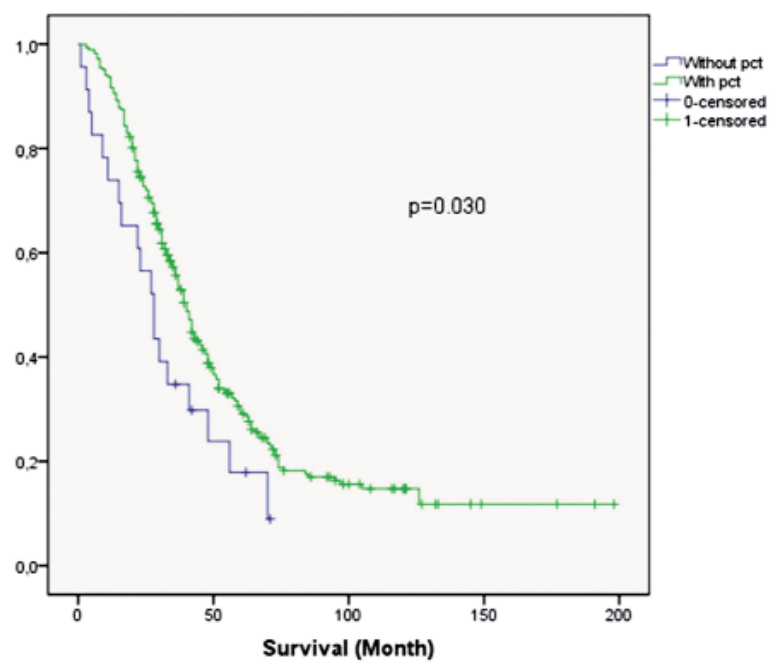

Figure 9 - Comparative overall survival between on Adjuvant Chemotherapy

after resection of CLMs it was performed a multivariate analysis. The multivariate Cox proportional hazards regression analysis was carried out by using the parameters that generated a $p$ value $<0.1$ at univariate analysis. Because of the very high number of patients with lack of data on CEA and CA 19-9 levels, these two parameters were not incorporated in multivariate analysis. Thus, the variables analyzed were: $\mathrm{T}$ status, $\mathrm{N}$ status (NO/N1 vs. N2), maximum diameter of metastases $(<5 \mathrm{~cm}$ vs. $>5 \mathrm{~cm}$.), number of CLMs $(<4$ vs. $\geq 4$ metastases), metastases distribution (uni- vs. bilobar), extent of hepatectomy, completeness of resection (RO vs. R1/R2), adjuvant chemotherapy and extrahepatic disease (table 2). 


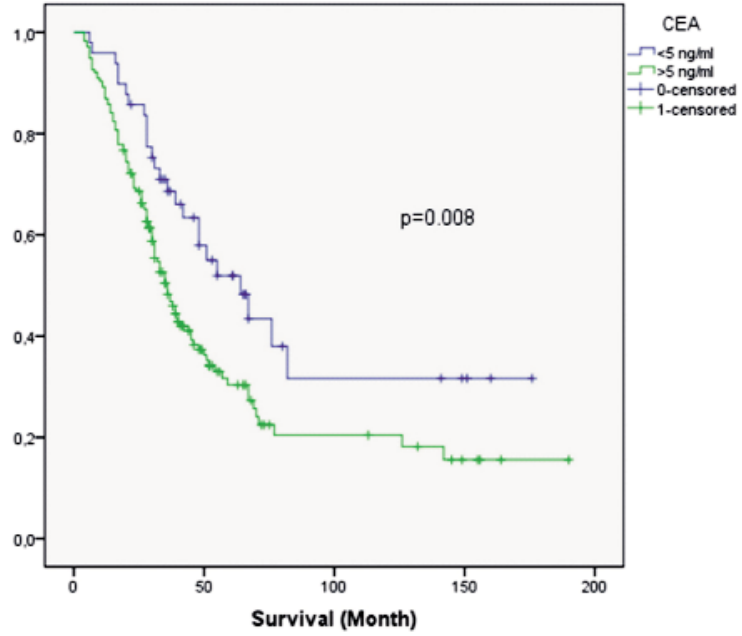

Figure 10 - Comparative survival based on CEA

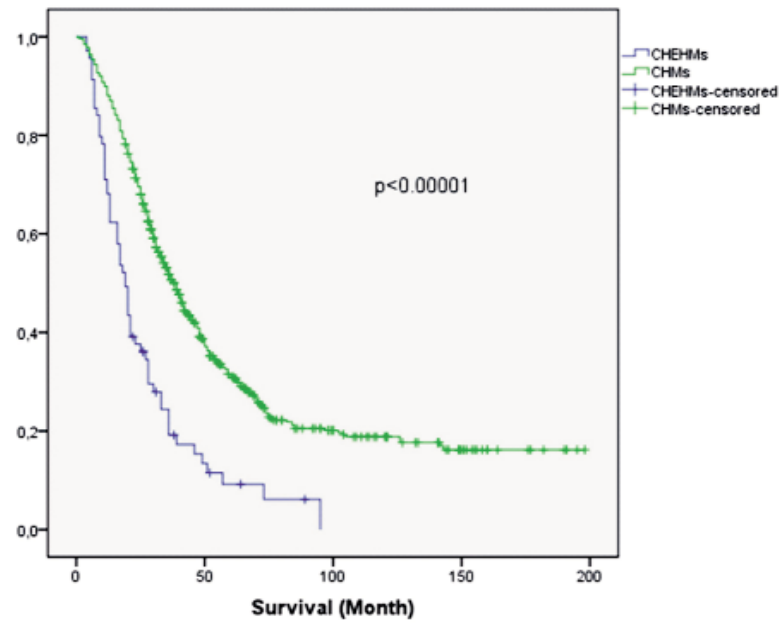

Figure 12 - Comparative overall survival CHEHMs vs CHMs

The independent prognostic factors for poor OS in patients who underwent resection of CLMs were: incomplete resection of metastases (R1/R2 - which increased the likelihood of death of 6 times), bilobar distribution of CLMs, 4 or more metastatic regional

Table 2 - Independent predictors for poor OS in patients who underwent liver resection for CLMs

\begin{tabular}{lcccc}
\hline & Sig. & HR & \multicolumn{2}{c}{$\mathbf{9 5 . 0 \%}$ Cl for Exp(B) } \\
\hline & & & Lower & Upper \\
\hline Bilobar liver metastasis & .003 & 1.653 & 1.180 & 2.316 \\
\hline R1/R2 liver resection & .000 & 6.066 & 3.508 & 10.489 \\
\hline N2 stage & .007 & 1.528 & 1.125 & 2.075 \\
\hline No adjuvant chemotherapy & .046 & 1.703 & 1.009 & 2.873 \\
\hline
\end{tabular}

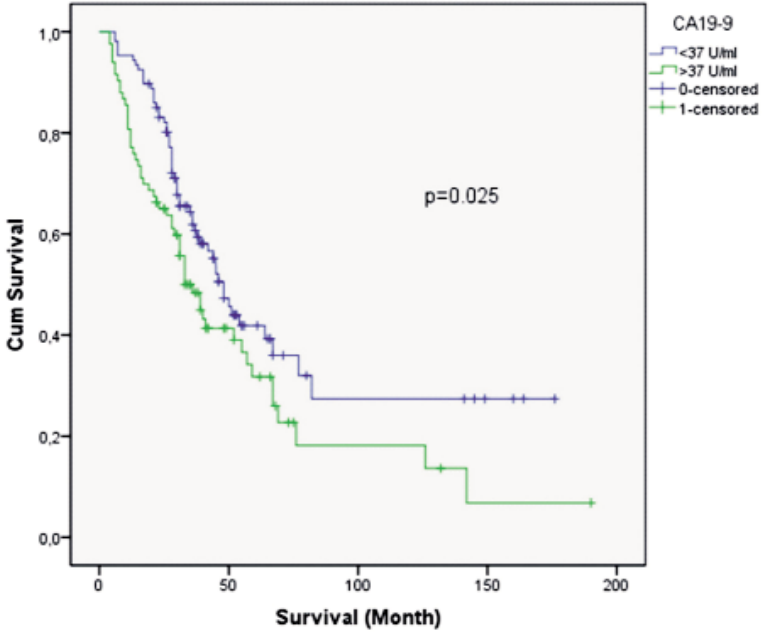

Figure 11 - Comparative overall survival based on CA19-9

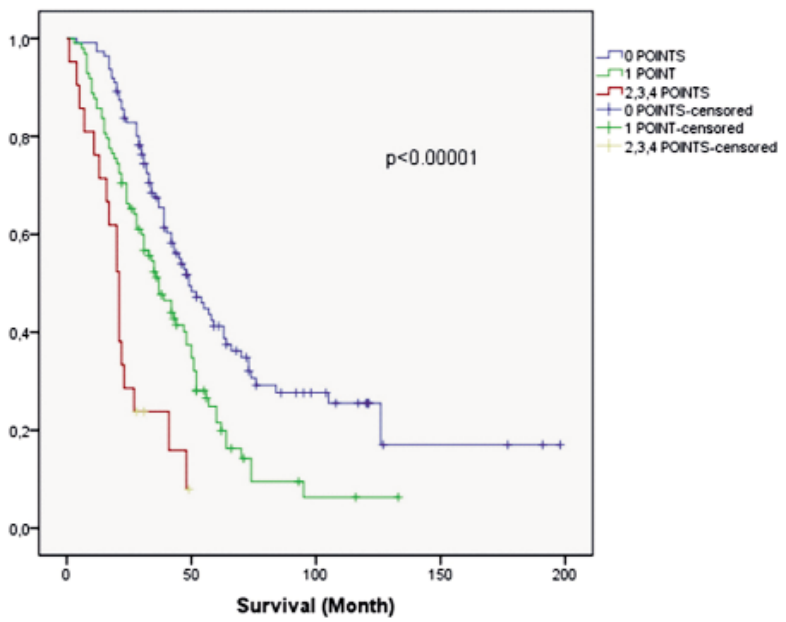

Figure 13 - Risk Score

lymph nodes (N2) and lack of adjuvant chemotherapy (increasing the risk of death of 1.65, 1.52 and 1.7 times, respectively).

Allocating 1 point to each of these independent prognostic factors, we stratified the patients in this cohort in 3 groups: group 0 - patients who did not present any of these poor prognostic factors, group 1 patients with only one prognostic factor for poor outcome and group 2 - patients presenting 2 to 4 prognostic factors. The median OS rates for group 0 , group 1 and group 2 were 49, 37 and 21 months, respectively ( $p$ value $<0.0001$ ) (Fig. 13). The survival analysis for these groups revealed that the combination of identified prognostic factors is able to accurately stratify the patients in terms of OS. 


\section{DISCUSSION}

Although the presence of liver metastases in patients with colorectal cancer represents an advanced stage of disease (stage IVA in 8th edition of AJCC staging system), the survival of these patients was significantly improved during the last three decades by the use of liver resection and oncologic therapy (cytotoxic agents and monoclonal antibodies)(2). Currently, it is universally accepted that liver resection is associated with the highest survival rates $(3,14-24)$. Even in the present study the 10 -year OS rate was $17.1 \%$, similar to those already reported in the literature $(10,14)$. However, the results of liver resection for CLMs are not uniform. There are patients who experienced prolonged survivals or even cure, as well as patients with very low OS rates. In such instances, many authors tried to discover the predictors of long term survival, to identify the patients who benefitted the most from liver resection. Moreover, the knowledge of prognostic factors helps in tailoring an individualized therapy.

The results of earlier studies were heterogeneous, with multiple clinico-pathologic factors associated with OS. Guided by these results we have also incorporated in our analysis most variables identified in previous studies $(10-13,15,25)$. Similar with earlier analyses, demographic variables such age and gender were not associated with OS in the present study. Unlike recent reports which revealed that right sided colon tumors were associated with poorer OS after resection of CLMs $(26,27)$, in the present analysis, the location of the primary did not significantly influence survival. Metastatic right sided tumors portend a more aggressive biology (than left-sided tumors), reflected mainly in more frequent hepatic and extrahepatic metastases and unresectable CLMs, but the influence of the primary tumor sidedness in patients with resectable CLMs was not proved in our series. The other variables related to the colorectal tumor ( $\mathrm{T}$ status, $\mathrm{N}$ status) significantly impact OS in univariate analysis, but only the presence of 4 or more metastatic lymph nodes (N2) was a significant independent prognostic factor for lower OS. These results are similar to those reported by Yamaguchi (28). The other scoring systems (10-13), use as independent prognosis of poor survival the presence of regional lymph node metastases, irrespective their number, undiscriminating between $\mathrm{N} 1$ and $\mathrm{N} 2$ patients. Nordlinger and Nagashima found that T3 and T4 colorectal tumors were independent prognostic factors for decreased OS, but the percentage of $\mathrm{T} 1 / \mathrm{T} 2$ patients in their studies (similar with this study) was very low $(11,12)$.
The timing of CLMs development (synchronous with the primary tumor or metachronous) has no significant influence on survival, although Konopke et al. identified the synchronous CLMs as independent prognostic of poor survival (13) and incorporated this parameter in their clinical scoring system. Moreover, synchronous metastases could be seen as a surrogate of poor prognosis in the studies of Nordlinger and Fong (they found disease-free interval $<24$ or 12 months, respectively, as independent predictor for decreased survival) $(10,11)$. However, the group from Fundeni Clinical Institute reported similar OS rates between patients undergoing liver resection for synchronous vs. metachronous CLMs, since 2009 (9,29). In previous studies, initial resectability came up as prognostic factor for better survival in univariate analysis $(8,30-32)$, but no one of the scoring systems elaborated until now includes this parameter as independent prognostic factor for OS. In the present analysis, the OS of initially unresectable patients rendered to resectability was not statistically significant lower than in initially resectable CLMs group (univariate analysis).

In contrast with most studies dealing with the subject of prognostic factors after CLMs resection, in the present series, the only parameter related to the liver metastases that independently influences OS was bilobar distribution, which increased the likelihood of death of 1.65 times. The bilobar distribution was also introduced as prognostic factor in MSKCC nomogram (33), but the other scoring systems did not incorporate this factor as prognostic for poor OS $(10-13,28,34,35)$. The number of CLMs (cut-off value of 4), as well as their maximum diameter (cut-off value of $5 \mathrm{~cm}$ ) were not identified as independent prognostic factors in multivariate analysis in this study, although 4 or more CLMs and metastases larger than $5 \mathrm{~cm}$ were significantly associated with lower OS in univariate analysis. This finding may be explained by the frequent use in this cohort of modern adjuvant chemotherapy and targeted therapies (in patients who recurred after liver resection), which may have more impact on survival than traditional parameters related to CLMs (36).

Unlike the previous studies which incorporated in analysis only clinico-pathologic variables, the present study also evaluated the effect of the factors related to the surgery and adjuvant therapies (that may be correlated with survival). The factor that most influenced the OS was related to the surgery, with R1/R2 resections increasing 6-fold the risk of death. Similar results were reported in most studies (10,37-39), although recent papers suggested that the results of R1 resection are mitigated in present by the effective oncologic therapies 
(40-45). However, prospective R2 resections are still an absolute contraindication to liver resection for CLMs and whenever possible, a negative resection margin larger than $1 \mathrm{~mm}$ should be achieved $(36,46-48)$. To evaluate the potential benefit offered by preoperative (neoadjuvant) or postoperative (adjuvant) chemotherapy in patients undergoing hepatectomy for CLMs, this study also evaluated the effect of these two parameters. Neoadjuvant chemotherapy had no impact on OS, but adjuvant chemotherapy significantly improved survival, both in univariate and multivariate analysis. Thus, the lack of adjuvant oncologic treatment increases the likelihood of death 1.7-fold. Identification of the adjuvant chemotherapy as independent prognostic factor for prolonged OS supports the above mentioned hypothesis that modern effective chemotherapy mitigates the traditional tumor and patient characteristics correlated with poor survival. The influence of better surgery and evolving oncologic therapies on CLMs patients' survival is also reflected in changing trends of OS over the time period of the study. Thus, during the initial period (1996-2005) the liver surgery program was implemented and developed in "Dan Setlacec" Centre of General Surgery and Liver Transplantation from Fundeni Clinical Institute and in most patients who received chemotherapy the regimens consisted in 5 -Fluorouracil (5-FU); the higher number of patients without chemotherapy was also recorded in this time period. Consequently, the median survival rate was 29.4 months (the lowest among the three periods). The better OS (median survival 33.9 months) during the second period (2006-2010) could be attributed to the high level of expertise achieved in hepatic surgery and to the introduction on a large scale of doublet chemotherapy regimens (5-FU + Oxaliplatin/Irinotecan). The statistically significant increase in OS (median survival 39.4 months, $p$ value $=0.001$ ) achieved in the last period (2011-2016) could be mainly explained by the advent of targeted therapies (mainly Bevacizumab and Cetuximab).

Surprisingly, unlike most studies(3, 10, 49-52), in this study, the presence of extrahepatic disease was not found as an independent prognostic of poor OS; although in univariate analysis, the OS rates of patients with concomitant extrahepatic disease were highly significant lower than in patients with liver-limited disease ( $p$ value $<0.0001$ ). This fact could be explained by the higher proportion of patients with extrahepatic disease who underwent R1/R2 resections (28.9\%), that may induce a bias in independent analysis of this parameter (the effect of the presence of extrahepatic disease could be mitigated by the prognostic effect of incomplete resection). However, our results confirm that the concomitant presence of extrahepatic disease (stage IVB $-7^{\text {th }}$ edition of AJCC staging system) is no longer an absolute contraindication of hepatectomy in such patients, in prospect of complete resection of tumor burden (RO resection). Authors from high volume centres specialized in oncologic surgery provided similar recommendations during the last decade $(14,52-57)$.

The four independent prognostic factors were incorporated in a risk scoring system by attributing 1 point each. Thus, the risk score ranges from 0 to 4 . The patients who did not present any of these factors were included in group 0 (risk score -0 points), the patients with one prognostic factor were included in group 1 (risk score -1 point) and the patients who cumulated at least two prognostic factors form the group 2 (risk score - 2-4 points). The difference in OS rates among the three groups (compared by log-rank test) was highly statistical significant ( $p$ value $<0.0001$ ). Thus, this risk scoring system is able to stratify the patients in 3 risk groups ( 0 points - low risk; 1 point - intermediate risk; 2-4 points - high risk). Although criticisms may be addressed to the incorporation of the therapeutic parameters (R1/R2 resection and adjuvant chemotherapy) in a scoring system, the intention of this study was not to release a clinical risk score system, but evaluate how clinico-pathologic factors and therapeutic approaches influence OS in patients with CLMs. Thus, in patients with favourable clinico-pathologic prognostic factors (NO/N1 and/or unilobar distribution of CLMs group 0/group 1) the prognosis is tremendously influenced by an adequate therapeutic strategy. In such patients, high quality surgery (RO resection) and appropriate oncologic treatment is able to achieve significantly prolonged OS rates (specific to the patients from group 0/1), whereas R1/R2 resection and/or inaccurate oncologic therapy will translate in poor OS (corresponding with survival rates achieved in group 2). By contrary, in patients with poor clinico-pathologic prognostic factors (N2 and bilobar CLMs), the prognosis is mostly influenced by tumor biology and seems to be marginally influenced by therapy (0\% 5 -year OS rates).

Beside the clinico-pathologic factors identified in most studies, which may stratify patients and contribute to the tailoring of oncologic therapy, recent research has focused on using molecular and genetic biomarkers to identify subgroups of patients most likely to derive benefit from a given therapy and using better estimates of survival to guide individualized patient treatment plans(36, 58-60). Adding such biomarkers to the established prognostic clinic and 
pathologic factors might translate in improved survival rates in specific groups of patients with CLMs.

There are some limitations of our study that we had to consider. Firstly, because the study is retrospective, explained significant amount of missing data for some variables (i.e. CEA, CA 19-9) that ultimately led to their exclusion from the panel used in the multivariate analysis. Secondly, the long duration of enrolment included different periods that had some changes like chemotherapy (from nothing, to 5FU, Oxaliplatin and more recently Bevacizumab or Cetuximab). For this reason we have analyzed only presence or absence of chemotherapy and did not take in account the type of chemotherapy. Thirdly, because all the included patients in our study were Caucasian, it is possible that these findings may not translate to patients of other ethnicities.

\section{CONCLUSION}

In conclusion, this retrospective study (dealing with patients who underwent liver resection for CLMs) revealed that the independent prognostic factors for poor OS were both clinico-pathologic (4 or more metastatic regional lymph nodes - N2 and bilobar distribution of CLMs) and therapeutic (incomplete resection - R1/R2 and lack of adjuvant chemotherapy). These factors are able to prognostic stratify the patients undergoing liver resection for CLMs. In patients with good prognostic factors, an appropriate onco-surgical treatment is of paramount importance to significantly prolong survival, whereas in patients with poor prognostic factors, the tumor biology is less influenced by current oncologic therapies.

\section{REFERENCE}

1. Siegel RL, Miller KD, Jemal A. Cancer statistics, 2016. CA Cancer J Clin. 2016;66(1):7-30.

2. Kopetz S, Chang GJ, Overman MJ, Eng C, Sargent DJ, Larson DW, et al. Improved survival in metastatic colorectal cancer is associated with adoption of hepatic resection and improved chemotherapy. J Clin Oncol. 2009;27(22):3677-83.

3. Hughes KS SR, Songhorabodi S, Adson MA, Ilstrup DM, Fortner JG et al. Resection of the liver for colorectal carcinoma metastases: a multi-institutional study of patterns of recurrence. Surgery. Surgery. 1988:278-88.

4. Adson MA vHJ, Adson MH, et al. Resection of hepatic metastases from colorectal cancer. Arch Surg. 1984:647-51.

5. Choti MA SJ, Tiburi MF, Sumetchotimetha W, Rangsin R, Schulick RD, et al. Trends in long-term survival following liver resection for hepatic colorectal metastases. Ann Surg. 2002:759-66.

6. Scheele J A-HA. Resection of colorectal liver metastases. Langenbecks Arch Surg 1999:313-27.

7. Jaeck D BP, Guiguet M, Boudjema K, Vaillant JC, Balladur P, et al. Long-term survival following resection of colorectal hepatic metastases. Association Francaise de Chirurgie Br J Surg. 1997:977-80.
8. Eddie K. Abdalla M, Jean-Nicolas Vauthey, MD, Lee M. Ellis, MD, Vickie Ellis, Raphael Pollock, MD, PhD, Kristine R. Broglio, Kenneth Hess, $\mathrm{PhD}$, and Steven A. Curley, MD. Recurrence and Outcomes Following Hepatic Resection, Radiofrequency Ablation, and Combined Resection/Ablation for Colorectal Liver Metastases. Ann Surg 2004:818-27.

9. Sorin Alexandrescu, Andrei Diaconescu, Razvan Grigorie, Zenaida Ionel, Doina Hrehoret, Vladislav Brasoveanu, Mihnea Ionescu, Irinel Popescu. Surgical treatment of colorectal liver metastases - a single center experience over 20 years. J Transl Med Res. 2015:222-32.

10. Fong Y FJ, Sun RL, Brennan MF, Blumgart LH. Clinical score for predicting recurrence after hepatic resection for metastatic colorectal cancer: analysis of 1001 consecutive cases. Ann Surg 1999:309-18.

11. Nordlinger B GM, Vaillant JC, Balladur P, Boudjema K, Bachellier P, et al. Surgical resection of colorectal carcinoma metastases to the liver. A prognostic scoring system to improve case selection, based on 1568 patients. . Association Française de Chirurgie. 1996:1254-62.

12. Nagashima I TT, Adachi M, Nagawa H, Muto T, Okinaga K. Proposal of criteria to select candidates with colorectal liver metastases for hepatic resection: comparison of our scoring system to the positive number of risk factors. World J Gastroenterol. 2006:6305-9.

13. Konopke R KS, Distler M, et al. Prognostic factors and evaluation of a clinical score for predicting survival after resection of colorectal liver metastases. Liver Int. 2009:89-102.

14. Scheele J SR, Altendorf-Hofmann A, Paul M. Resection of colorectal liver metastases. World J Surg 1995:59-71.

15. Gayowski TJ IS, Madariaga JR, Selby R, Todo S, Irish W, Starzl TE. Experience in hepatic resection for metastatic colorectal cancer: analysis of clinical and pathologic risk factors. Surgery. 1994:710-1.

16. Rosen CB ND, Taswell HF, et al. Perioperative blood transfusion and determinants of survival after liver resection for meastatic colorectal carcinoma. Ann Surg. 1992:492-505.

17. Nordlinger B PR, Delva E, Quilichini M, Hannoun L, Huguet C. Hepatic resection for colorectal liver metastases. Ann Surg. 1987:256-63.

18. Fong Y CA, Fortner JG, et al. Liver resection for colorectal metastases. J Clin Oncol 1997:938-46.

19. Cobourn CS ML, Langer B, Taylor B, Falk R. Examination of patient selection and outcome for hepatic resection for metastatic disease. Surg Gynecol Obstet. 1987:239-46.

20. Schlag P HP, Herfarth C. Resection of liver metastases in colorectal cancer: competitive analysis of treatment results in synchronous versus metachronous metastases. s Eur J Surg Oncol 1990:360-5.

21. Doci R GL, Bignami P, Montalto F, Morabito A, Bozetti F. One hundred patients with hepatic metastases from colorectal cancer treated by resection: analysis of prognostic determinants. Br J Surg. 1991:797801.

22. Younes RN RA, Brennan MF. The influence of intraoperative hypotension and perioperative blood transfusion on disease-free survival in patients with complete resection of colorectal liver metastases. Ann Surg 1991. 1991:107-13.

23. Scheele J SR, Altendorf-Hofmann A, Gall FP. Indicators of prognosis after hepatic resection for colorectal secondaries. Surgery. 1991:13-29.

24. Cady B SM, McDermott WV Jr, et al. Technical and biological factors in disease-free survival after hepatic resection for colorectal cancer metastases. Arch Surg. 1992:561-9.

25. Cady B SM. The role of surgical resection of liver metastases in colorectal carcinoma. Semin Oncol. 1991:399-406.

26. Sasaki K AN, Margonis GA, He J, Weiss M, Johnston F, et al. The prognostic implications of primary colorectal tumor location on recurrence and overall survival in patients undergoing resection for colorectal liver metastasis. J Surg Oncol 2016:803-9.

27. Yamashita S BK, Kopetz SE, Maru D, Clarke CN, Passot G, et al. Embryonic Origin of Primary Colon Cancer Predicts Pathologic Response and Survival in Patients Undergoing Resection for Colon Cancer Liver Metastases. . Ann Surg 2016.

28. Yamaguchi T MT, Takahashi K, Matsumoto H, Miyamoto H, Kato T. A new classification system for liver metastases from colorectal cancer in Japanese multicenter analysis. Hepatogastroenterology. 2008:173-8.

29. Popescu I, Alexandrescu S, Dima S, Ionescu M, Hrehoret D, Brasoveanu V. Synchronous vs Metachronous Colorectal Liver Metastases and Timing of Liver Resection - Do They Influence Patients' 
Outcome? GASTROENTEROLOGY. 2009;136:815

30. Adam R DV, Pascal G, Valeanu A, Castaing D, Azoulay D et al. Rescue surgery for unresectable colorectal liver metastases downstaged by chemotherapy: a model to predict long-term survival. Ann Surg 2004:644-57.

31. Chun YS VJ. Extending the frontiers of resecabilyty in advanced colorectal cancer. Eur J Surg Oncol. 2007:52-8.

32. S Giacchetti MI, G Gruia, et al. Long-term survival of patients with unresectable colorectal cancer liver metastases following infusional chemotherapy with 5-fluorouracil, leucovorin, oxaliplatin and surgery. Ann Oncol 1999:663-9.

33. Kattan MW GM, Jarnagin WR, DeMatteo R, D'Angelica M, Weiser M, et al. A nomogram for predicting disease-specific survival after hepatic resection for metastatic colorectal cancer. Ann Surg. 2008:282-7.

34. Rees M TP, Welsh FK, O'Rourke T, John TG. Evaluation of longterm survival after hepatic resection for metastatic colorectal cancer: a multifactorial model of 929 patients. Ann Surg. 2008:125-35.

35. Damm R SR, Ulrich G, Breier L, Steffen IG, Seidensticker M, et al. Y90 Radioembolization in chemo-refractory metastastic, liver dominant colorectal cancer patients: outcome assessment applying a predictive scoring system. BMC Cancer. 2016.

36. Jeffery Chakedis MHS, Eliza W. Beal, Tasha Hughes, Heather Lewis, Anghela Paredes,Mazen Al-Mansour, Steven Sun, Jordan M. Cloyd, Timothy M. Pawlik. Update on Current Problems in Colorectal Liver Metastasis. Current Problems in Surgery. 2017.

37. Minagawa M YJ, Miwa S, Sakamoto Y, Kokudo N, Kosuge T et al. Selection criteria for simultaneous resection in patients with synchronous liver metastasis. Arch Surg 2006:1006-12.

38. Sugawara Y YJ, Yamasaki S, Shimada K, Kosuge T, Makuuchi M Estimating the prognosis of hepatic resection in patients with metastatic liver tumors from colorectal cancer with special concern for the timing of hepatectomy. Surgery 2001:408-13.

39. Fujita S AT, Moriya Y. Resection of synchronous liver metastases from colorectal cancer. Jpn J Clin Oncol 2000:30(1):7-11.

40. de Haas RJ WD, Flores E, Azoulay D, Castaing D, Adam R. R1 resection by necessity for colorectal liver metastases: is it still a contraindication to surgery? Ann Surg 2008:627-37.

41. Alexandrescu S, Diaconescu A, Ionel Z, Zlate C, Grigorie R, Hrehoret D, et al. Comparative analysis between simultaneous resection and staged resection for synchronous colorectal liver metastases - A single center experience on 300 consecutive patients. Chirurgia 2017.

42. Andreou A AT, Brouquet A, Dickson PV, Zimmitti G, Maru DM, et al: Margin status remains an important determinant of survival after surgical resection of colorectal liver metastases in the era of modern chemotherapy. Ann Surg 2013:1079-88.

43. Margonis GA SG, Kim Y, Ejaz A, Pawlik TM. Intraoperative surgical margin reresection for colorectal liver metastasis: is it worth the effort? J Gastrointest Surg. 2015:699-707.

44. Botea F IM, Brasoveanu V, Hrehoret D, Alexandrescu S, Grigorie M, Stanciulea 0, Nicolaescu D, Tomescu D, Droc G, Ungureanu D, Fota R, Croitoru A, Gheorghe L, Gheorghe C, Lupescu I, Grasu M, Boros M, Dumitru R, Toma M, Herlea V, Popescu I. Liver Resections in a HighVolume Center: Form Standard Procedures to Extreme Surgery and Ultrasound-guided Resections. Chirurgia. 2017:259-77.

45. Viganò L PF, Cimino MM, Donadon M, Gatti A, Costa G, et al. Is tumor detachment from vascular structures equivalent to $r 0$ resection in surgery for colorectal liver metastases? An observational cohort. Ann Surg Oncol. 2016:1352-60.
46. Pawlik TM SC, Zorzi D, Abdalla EK, Andres A, Eng C, Curley SA, Loyer EM, Muratore A, Mentha G. Effect of surgical margin status on survival and site of recurrence after hepatic resection for colorectal metastases. Anna Surg 2005:715-24.

47. Viganò $L$ CL, Lapointe $R$, et al. Early recurrence after liver resection for colorectal metastases: Risk factors, prognosis, and treatment. A LiverMetSurvey-based study of 6,025 patients. Ann Surg Oncol. 2014:1276-86.

48. Sadot E GKB, Leal JN, Shia J, Gonen M, Allen PJ, et al. Resection margin and survival in 2368 patients undergoing hepatic resection for metastatic colorectal cancer: surgical technique or biologic surrogate? Ann Surg 2015:483-5.

49. Ekberg H TK, Andersson R, Lundstedt C, Hagerstrand I, Ranstam J et al. Determinants of survival in liver resection for colorectal secondaries. Br J Surg 1986:727-31.

50. Stangl R A-HA, Charnley RM, Scheele J. Factors influencing the natural history of colorectal liver metastases. Lancet. 1994:1405-10.

51. Adam R HD, Poston G, Raoul JL, Spano JP, Tabernero J, Van Cutsem E. Toward optimized front-line therapeutic strategies in patients with metastatic colorectal cancer--an expert review from the International Congress on Anti-Cancer Treatment (ICACT) Ann Oncol. 2010:1579-84.

52. Minagawa M MM, Torzilli G, Takayama T, Kawasaki S, Kosuge T et al. Extension of the frontiers of surgical indications in the treatment of liver metastases from colorectal cancer: long-term results. Ann Surg. 2000:487-99.

53. Nordlinger B JD, Balladur P, Vaillant JC, Paris F. Surgical resection of hepatic metastases. Comments on the results of the multicentric retrospective study by the French Association of Surgery. In: Nordlinger B, Jaeck D, editors Treatment of hepatic metastases of colorectal cancer. 1992:147-57.

54. Carpizo DR AC, Jarnagin W, DeMatteo R, Fong Y, Gonen M et al. Liver resection for metastatic colorectal cancer in patients with concurrent extrahepatic disease: results in 127 patients treated at a single center. Ann Surg Oncol 2009:2138-46.

55. William J. Hadden PRdR, Kai Brown, Anubhav Mittal, Jaswinder S. Samra, and Thomas J. Hugh. Resection of colorectal liver metastases and extra-hepatic disease: a systematic review and proportional metaanalysis of survival outcomes. HPB ( Oxford). 2016.

56. Pulitano C. BM, Aldrighetti L., Choti M.A., Castillo F., Schulick R.D. Colorectal liver metastasis in the setting of lymph node metastasis: defining the benefit of surgical resection. Ann Surg Oncol. 2012:43542.

57. Oussoultzoglou E. RB, Panaro F., Rosso E., Pessaux P., Bachellier P. Long-term survival after liver resection for colorectal liver metastases in patients with hepatic pedicle lymph nodes involvement in the era of new chemotherapy regimens. Ann Surg. 2009.

58. Brudvik KW KS, Li L, Conrad C, Aloia TA, Vauthey JN. Meta-analysis of KRAS mutations and survival after resection of colorectal liver metastases. Br J Surg 2015:175-83.

59. Mise Y ZG, Shindoh J, Kopetz S, Loyer EM, Andreou A, et al:. RAS mutations predict radiologic and pathologic response in patients treated with chemotherapy before resection of colorectal liver metastases. Ann Surg Oncol 2015:834-42.

60. Schirripa M BF, Cremolini C, Casagrande M, Lonardi S, Aprile G, et al. BRAF and RAS mutations as prognostic factors in metastatic colorectal cancer patients undergoing liver resection. $\mathrm{Br} \mathrm{J}$ Cancer 2015:1921-8. 